\title{
Method for Spot-Detection on Solar-Like Stars
}

\author{
Adriana V. R. Silva \\ Universidade Presbiteriana Mackenzie, CRAAM, \\ Rua da Consolação, 896, São Paulo, SP 01302-907, Brazil
}

\begin{abstract}
As a planet eclipses its parent star, a dark starspot may be occulted, causing a detectable variation in the light curve. This work describes how features on the surface of a solar-like star can be studied by using planetary transits. Images of the Sun were used in order to simulate such transits, with the planet being modeled as a dark disk at various positions along its orbit. From modeling of these transits it might be possible to infer the physical properties of the spots, such as size, intensity, position, and temperature. Recent transits observations for HD 209458 were used as tests to the model. The results yield that the limb darkening of HD 209458 is not like that of the Sun, but rather follows a quadratic function. The dark features studied have radii varying between $3-610^{4} \mathrm{~km}$, and probably represent a group of spots, namely an active region. As for the temperature, these spots are hotter than sunspots with a temperature range of $4900-5500 \mathrm{~K}$.
\end{abstract}

\section{Introduction}

Over the centuries, since their discovery by Galileo, sunspots have been monitored almost daily. It is now known that these dark features on the surface of the Sun are regions of higher concentration of magnetic field. Sunspots are also a good indicators of the solar activity, which has a periodicity of 11 years. Similarly, solar-like stars are likely to also have regions of enhanced magnetic field and even follow some sort of magnetic cycle. Thus, the studies of these starspots will provide important clues to the stellar magnetic activity.

One way to study these features is through their eclipse by a transiting planet around the host star. Spots like those on the Sun are very small and would only be a few microarcsec even for the nearest stars, rendering their direct detection impossible. Examples of planetary transits on our own Solar System are the recent transit of Mercury on May 7th, 2003 (Figure 1) and the upcoming Venus transit on June 8th of 2004.

Presently, over a hundred stars are known to harbor planets in orbit around them (Extrasolar Planets Encyclopaedia, www.obspm.fr/planets). The detection of planets was achieved by the small radial velocities variations observed in the stellar spectral lines, and interpreted as due to the gravitational pull of a massive planet close in (Mayor \& Queloz 1995, Butler et al. 1997). The first confirmation of the existence of such a planet came from the detection of 


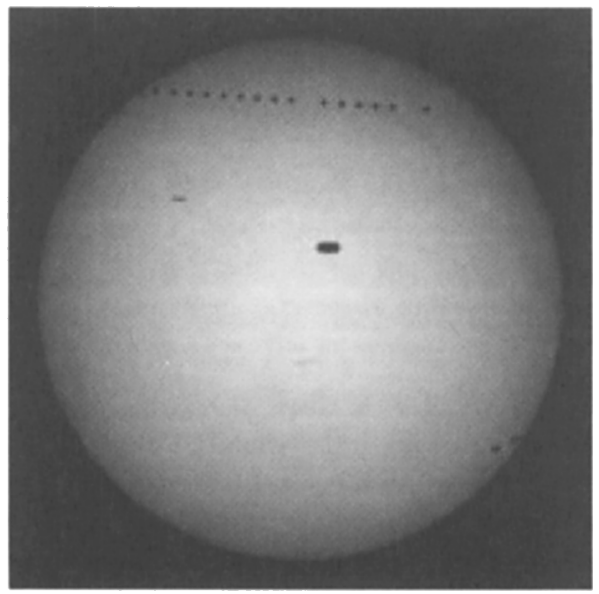

Figure 1. Composite images of Mercury transit on May 7th, 2003, taken by the GONG Network from India.

a dimming in the light curve of HD 209458 (Jha et al. 2000), explained by the transit of the planetary companion. The transits of HD 209458b have been extensively studied in the literature (Henry et al. 2000, Charbonneau et al. 2000, Jha et al. 2000, Brown et al. 2001, Deeg et al. 2001) and the physical parameters of the host star and its planetary companion are well established (Cody and Sasselov, 2020), as well as the orbital parameters.

The main goal of this work is to establish a method for the characterization of stellar spots through the observation of planetary transits (Silva, 2003). The first star known to have a transiting planet, HD 209458, is a good candidate for this study in the sense that the planetary transit occurs at a stellar latitude of approximately $30^{\circ}$. In the case of the Sun, this is within the royal zones, that is, the regions were sunspots occur. The next section presents simulations of the transit by planets of various sizes relative to the solar-like spots. The model is described in Section 3, and tested on the observations of the HD 209458 transits on Section 4. Finally, in the last section, the results are discussed and the conclusions listed.

\section{Planetary transit simulations}

In order to estimate the effect of a planetary transit in front of a dark feature on the surface of a star, such as a sunspot, the transit of a planet was simulated using a white light image of the Sun from Big Bear Solar Observatory. Such image of the Sun can be seen in Figure 2 (left), where an active region, that is a group of sunspots is seen in the middle of the Northern hemisphere. Two simulations were performed in which a planet the size of the Earth and one the size of HD 209458 were made to cross the disk of the Sun along the dashed line. The relative sizes of the planetary disks are shown as dark circles in the figure. The results from the simulation can be seen in the right panels of Figure 2 , where the top panel shows the result for a terrestrial size planet, whereas the bottom panel displays the result for HD 209458. As can be seen from the figure, the effect of the eclipse of a dark feature such as a sunspot is to increase 

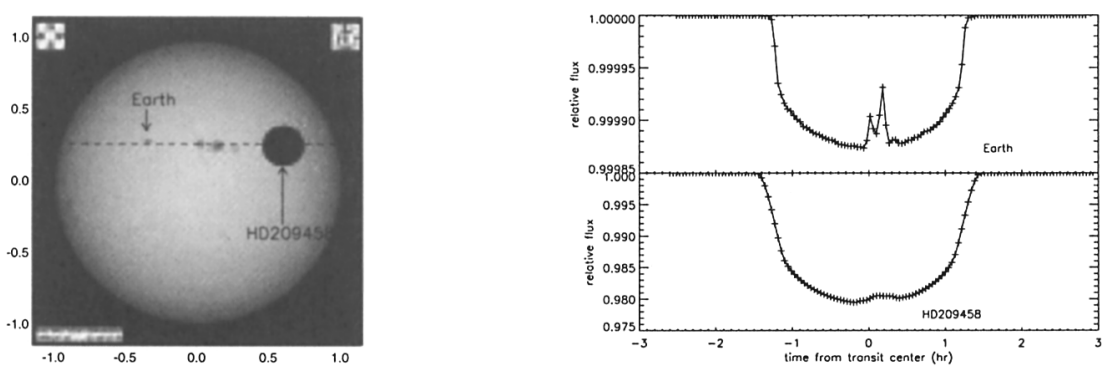

Figure 2. Left: Simulation of a transit across the disk of the Sun, for a planet the size of the Earth (left arrow) and the size of Jupiter (right arrow). Right: Effect of the eclipse of a planet over a sunspot for an Earth sized planet (top panel) and for a planet the size of HD 209458 (bottom panel).

the light flux momentarily. The effect of a planet the size of Earth as it passes in front of the spot relative to the total decline in intensity is larger than that caused by a planet larger than Jupiter. Moreover, even some structure may be identified. This happens because the size of the Earth is of the order of the sunspot. Nevertheless, the total decrease in intensity caused by the planetary transit is 100 times smaller for the case of an Earth sized planet compared to that of Jupiter.

\section{Model of planetary transit in front of a stellar spot}

Similarly to the simulations above, an exoplanet may also pass in front of a stellar group of spots during one of its transits. Here, a method for studying the physical characteristics of the starspot - such as size, temperature, and location - is proposed (Silva 2003). Observations of HD 209458 are used as test to this model (section 4). The stellar disk is represented by an image of the Sun (see Figure 2, left panel).

In the model, the planet around HD 209458 was assumed to be in a circular orbit, with a period of 3.5247 days (Henry et al. 2000), major semi-axis of 0.0467 $\mathrm{AU}$, and inclination angle, $\mathrm{i}=86.68$. The planet radius was $1.347 \mathrm{R}_{\mathrm{Jup}}$, and the stellar radius, $1.146 R_{\text {Sun }}$. As in the previous section, the planet is represented by an opaque disk that crosses the stellar disk at 30.45 latitude (corresponding to $\mathrm{i}=86.68$ ). Every two minutes, the planet position is calculated, and the pixels of the solar image (representing the star) corresponding to the planetary disk centered on these positions are replaced by zeros. The intensity of the lightcurve, with two minutes temporal resolution, is the sum of all the pixels values in the image.

As mentioned above, the starspot is modeled by three parameters: (a) intensity, as a function of stellar intensity at disk center (maximum); (b) size, as a function of planet radius; and (c) position, as a distance to the transit line in units of planet radius. The ideia is to fit the observed "bumps" detected in 

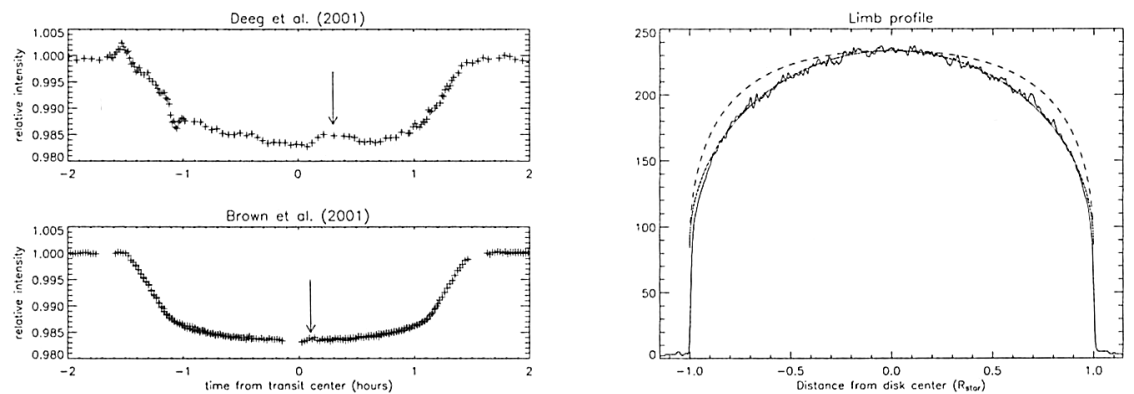

Figure 3. Left: Lightcurves of HD 209458 transits with "bumps indicated by arrows. Right: Intensity profile of the Sun as a function of distance from disk center. Also shown in the figure are the linear and quadratic limb darkening functions used by Brown et al. (2001).

the lightcurve of transits, as the one seen in the bottom right panel of figure 2, according to these three parameters.

\section{HD 209458 transits as a test to the model}

Since many transits of HD 209458 have been reported in the literature, transit lightcurves with variations were sought. Two such data obtained on April 25, 2000 using HST, from Brown et al. (2001), and on July 26, 2000 by the telescope of the Observatorio Sierra Nevada (Deeg et al. 2001) were selected. These lightcurves are displayed on Figure 3.

The Deeg et al. (2001) observations were fit by a set of parameters for the modeled starspots as described in the previous section. The best fit to the data is shown as a solid line in Figure 4 (left panels). Table 1, middle column, lists the range of parameter values that yield the best fit to the data, where the planetary radius is $R_{p}=9.410 \mathrm{~km}$. The spot is represented by the light gray circle in the bottom left panel of Figure 4. In this case, the star was represented by a white image of the Sun.

Table 1. Best fit results for the stellar spots to the transit data.

\begin{tabular}{lcc}
\hline & 26 -jul-2000 & 25 -apr-2000 \\
\hline Radius $\left(R_{p}\right)$ & $0.4-0.6$ & $0.3-0.4$ \\
Intensity $\left(I_{m} x\right)$ & $0.4-0.6$ & $0.5-0.7$ \\
Distance to transit line $\left(R_{p}\right)$ & $0.5-0.8$ & $0.7-0.9$ \\
\hline
\end{tabular}

The HST data (Brown et al. 2001), however, is not well fit by modeling the star by a solar image, indicating that the limb darkening of HD 209458 is not a linear function of $\mu=\cos \theta$, as that of the Sun, instead it is best described by a quadratic function of $\mu$ :

$$
\frac{I(\mu)}{I(1)}=1-w_{1}(1-\mu)-w_{2}(1-\mu)^{2} .
$$



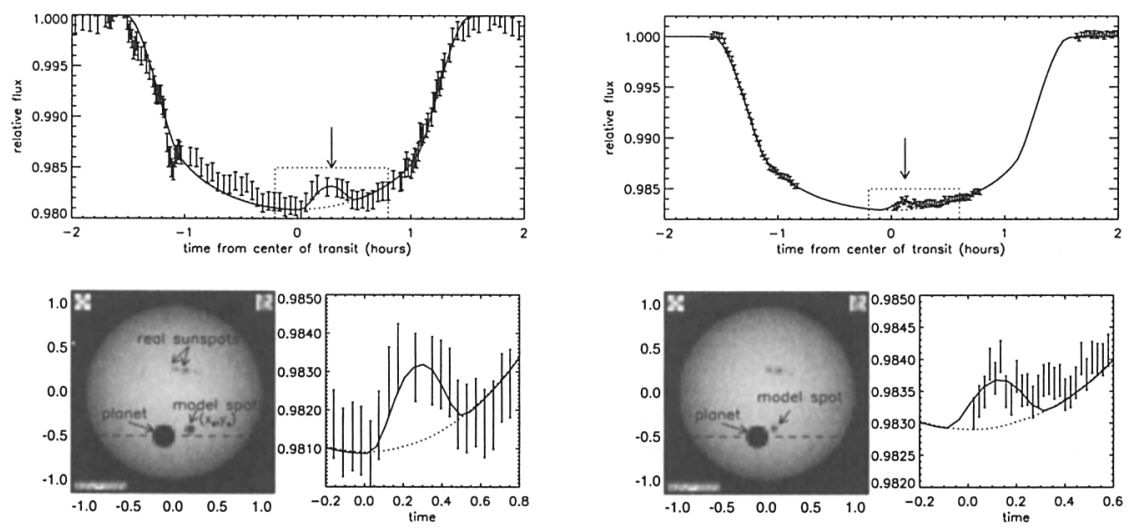

Figure 4. Left: Observations of Deeg et al. (2001) light curve fitted by the model (solid curve), whereas the dotted line represents a transit without spot occultation. A blow up of the box outlined in the top panel containing the "bump" due to the spot eclipse is shown below. Bottom left panel: image of the Sun used in the model with the opaque disk of the planet and the modeled spot (indicated by arrows). The dashed line shows the positions of the planetary transit used in the model. Right: Same as left panels for the observations made by the Hubble Space Telescope (Brown et al. 2001).

Figure 3, right panel, shows the difference of a linear (gray line) and a quadratic (dashed line) function of $\mu$, overlaid on a solar intensity profile, which is well described by a linear function of the limb darkening. Thus, in order to fit the HST data, a new model for the star was made with a quadratic limb darkening, with $w_{1}=0.2925$ and $w_{2}=0.3475$ (Brown et al. 2001). This discrepancy was not evident in the Deeg et al. data due to their larger uncertainty. The best fits to the HST lightcurve "bumps" are listed on the last column of Table 1 , and the best fit is plotted on the right panels of Figure 4. Note that these observations were fit by a smaller and darker spot.

\section{Discussion and conclusions}

The method presented here for analyzing planetary transits enables us to estimate the starspots physical parameters such as size, temperature, and location. The transit of HD 209458 was modeled from the values found in the literature (e.g., Brown et al. 2001). Recent transit data of HD 209458 with variations in the lightcurve were used in order to test the model. A good description of the data by the model was found for stellar spots with sizes of $3-610^{4} \mathrm{~km}$, being larger than regular sunspots, usually of the order of $11,000 \mathrm{~km}$. These structures, however, are probably a group of starspots, similar to solar active regions. 
The starspot temperature, $T_{0}$, may be estimated by considering blackbody emission, with an effective stellar surface temperature assumed to be $6000 \pm 50$ $\mathrm{K}$ (Mazeh et al. 2000). In this case, the spot intensities in the range of 40 to $70 \%$ of the star central intensity yield starspot temperatures between $4900-5000 \mathrm{~K}$. These estimated temperatures are hotter than regular sunspots (3800-4400K), however the surface temperature of HD 209458,6000K, is also hotter than that of the Sun $(5770 \mathrm{~K})$. Nevertheless, the sunspots seen in the white light image are also about $0.4-0.7$ of the solar disk center intensity, similarly to what was obtained from the model.

In the case of the Sun, sunspots are the primary and oldest indicators of magnetic activity. Similarly, from detailed long term study of spots detected by planetary transits, an understanding of stellar magnetic activity cycles may emerge based on the spots characteristics and frequency of occurrence, opening up a new research field. This method is proposed as a way to study stellar spots, since direct detection of such a small angular sized feature by photometric variations is not currently possible. Even more improbable is the spot direct observation by current or planned telescopes in the near future. Application of this method to observations from the COROT (Michel et al. 2000), Eddington (Favata, Roxburgh, \& Galadi), and Kepler (Koch et al. 1998) missions, especially designed for the purpose of detecting planetary transits, will definitely enhance our understanding of the magnetic activity on stars other than our Sun.

\section{References}

Brown, T. M., Charbonneau, D., Gilliland, R. L., Noyes, R. W., \& Burrows, A. 2001, ApJ, 552, 699

Butler, R. P., Marcy, G. W., Williams, E., Hauser, H., \& Shirts, P. 1997, ApJ, 474, L115

Charbonneau, D., Brown, T. M., Latham, D. W., \& Mayor, M. 2000, ApJ, 529, L45

Cody, A. M. \& Sasselov 2002, D. D., ApJ, 569, 451

Deeg, H. J., Garrido, R., \& Claret, A. 2001, New Astron., 6, 51

Favata, F., Roxbourgh, I. W., \& Galadi, D. 2001, Proc. of the First Eddington Workshop on Stellar Structure and Habitable Planet Finding, ESA pub. SP-485 (Cordoba, Spain)

Henry, G. W., Marcy, G. W., Butler, R. P., \& Vogt, S. S. 2000, ApJ, 529, L41

Jha, S., Charbonneau, D., Garnavich, P. M., Sullivan, D. J., Sullivan, T., Brown, T. M., \& Tonry, J. L. 2000, ApJ, 540, L45

Koch, D. G., Brorucki, W., Webstar, L., Dunham, E., Jenkins, J., Marriott, J., \& Reitsema, H. J. 1998, in Proc. SPIE, 3356, 599

Mayor M. \& Queloz, D. 1995, Nature, 378, 355

Mazeh, T. et al. 2000, ApJ, 532, L55

Michel, E. et al. 2000, in ASP Conf. Ser. 203, eds. L. Szabados \& D. Kurtz (ASP, San Francisco), 69

Silva, A.V.R. 2003, ApJ, 585, L147 